\title{
Good Deals and compatible modificatio of risk and pricing rule: a regulatory treatment
}

\author{
Hirbod Assa • Alejandro Balbás
}

\begin{abstract}
This work studies Good Deals in a scenario in which a fir uses decision-making tools based on a coherent risk measure, and in which the market prices are determined with a sub-linear pricing rule. The most important observation of this work is that the existence of a Good Deal is equivalent to the incompatibility between the pricing rule and the risk measure. In this paper, we look into this situation from a regulatory point of view to rule out Good Deals with the purpose of stabilizing financia markets. We propose some practical ways of modifying a risk measure so a regulator can set appropriate levels of capital requirements for a financia institution.
\end{abstract}

Keywords Coherent risk measure - Pricing rule - Good deal · Compatibility · Risk modificatio $\cdot$ Risk spread $\cdot$ Performance ratio

JEL Classificatio $\quad \mathrm{C} 020 \cdot \mathrm{G} 320$

\section{Introduction}

Inappropriate choice of risk measure may produce a type of pathological financia position we call a Good Deal, which is the major objective we will study in this paper. We study Good Deals when a decision-maker uses a coherent risk measure to assess the risk level of his/her financia position, and when the market values are given by a sub-linear pricing rule (in the sense of Jouini and Kallal $[15,16])$.

H Assa ( $\square)$

Department of Mathematics and Statistics \& GERAD, Université de Montréal,

Pavillon André-Aisenstadt 2920, chemin de la Tour, Montréal, QC, Canada

e-mail: assa@dms umontreal ca

A Balbás

Department of Business Administration, University Carlos III of Madrid, C/ Madrid,

126, 28903 Getafe, Madrid, Spain

e-mail: alejandro balbas@uc3m es 
Cochrane and Saà-Requejo [10] firs introduce the notion of a Good Deal as a financia position with particularly high Sharpe ratio. In this work, the authors assume that Good Deals do not exist in market equilibrium, and they show that this assumption holds if and only if there is a bound on the variance of the members of the Stochastic Discount Factor set (SDF). In Cochrane and Saà-Requejo [10], this problem is analyzed for the one-period, multi-period, and the continuous time settings. The definition of a Good Deal has been extended in Černý and Hodges [7], where the authors defin a set of "desirable" positions. They defin a Good Deal as a desirable position with the non-negative price and use the No Good Deal assumption to price the claims in an incomplete market. In another work, Černý [6] define a Good Deal by mean of a generalized Sharpe ratio, developing the ideas in Cochrane and Saà-Requejo [10] and Černý and Hodges [7].

Björk and Slinkor [5] extend the results of Cochrane and Saà-Requejo [10] to a dynamic setting with a general Markov process, allowing a study of the Good Deal bounds for processes with jumps. Cherny [9] extends the definition of Good Deals to positions with a high performance ratio (a generalization of Sharpe ratio). All these works aim to price a financia position in an incomplete market when equilibrium is reached.

Our work differs from the existing literature in two ways. First, we use our results for regulatory purposes (assessing the capital requirement), not for pricing. Second, we are interested in investigating a situation in which a Good Deal exists. We found that underestimating the risk of a financia position (or under-capitalization) produces Good Deals. To avoid undercapitalization, the financia institutions have to modify their risk measures to ones which always dominate the primary risk measures in use. In fact, the modifie risk measure must dominate the primary risk measure in addition to dominating the short selling price. In this paper, we propose two ways of modifying a risk measure. The firs regards the fundamentals of the risk user, and the second regards the fundamentals of the market. We also focus on concrete risk measures. Special attention is devoted to Conditional Value at Risk, CVaR, because this coherent risk measure has become very popular among researchers, managers and practitioners. We apply our finding to CVaR so as to build the Compatible Conditional Value at Risk (CCVaR) in a general incomplete market. We show that in an incomplete market, CCVaR can be found by seeking a stochastic discount factor with the smallest European call option price. This modifie the discussion in Balbás and Balbás [1], in which CCVaR is introduced in a complete perfect market (i.e. SDF is a singleton).

This paper is organized as follows. In Sect. 2, we will present the notations and the general framework with which we will work. We will consider an Arbitrage-free market (in general incomplete and/or imperfect) with a sub-linear pricing rule $\pi$ and a coherent risk measure $\rho$. In Sect. 3, we defin the concept of a Good Deal, inspired by definitions in Černý and Hodges [7] and Cherny [9]. We will show that incompatibility is equivalent to the existence of Good Deals. In Sect. 4, we will show the existence of a minimal compatible modificatio of a coherent risk measure. We will see that the existence of a minimal compatible modificatio is tied to the existence of a minimal point of a partial order on SDF. In Sect. 5, which constitutes the second part of the paper, we will propose two ways of modifying a risk measure.

\section{Preliminaries and notation}

Let $(\Omega, \mathcal{F}, \mathbb{P})$ be a probability space composed of the set $\Omega$ representing the"states of the nature", a $\sigma$-fiel $\mathcal{F}$ and a probability measure $\mathbb{P}$. Let $p, q \in[1, \infty]$ be two numbers such that $1 / p+1 / q=1$. For $p \neq \infty, L^{p}$ denotes the space of real-valued random variables $X$ on $\Omega$ such that $\mathbb{E}\left(|X|^{p}\right)<\infty$ where $\mathbb{E}$ represents the mathematical expectation. The space $L^{\infty}$ 
consists of all bounded random variables. Recall that according to the Riesz Representation Theorem, $L^{q}$ is the dual space of $L^{p}$ when $p \neq 1, \infty$. We endow the space $L^{p}\left(L^{q}\right)$ by two topologies, firs the norm topology and second the topology induced by $L^{q}\left(L^{p}\right)$ i.e. the coarsest topology in which all members of $L^{q}\left(L^{p}\right)$ are continuous. As usual the latter topology is called the weak topology and is denoted by $\sigma\left(L^{p}, L^{q}\right)$ (there is one exception for $p=\infty$ when $\sigma\left(L^{\infty}, L^{1}\right)$ is called weak star topology).

In this paper we consider only two periods of time, today and tomorrow, represented by 0 and $T$ respectively. Every random variable represents pay-off of a financia position at time $T$. Whenever we talk about risk or price of a financia position we mean the present value of the price and the present risk associated with the financia position. In addition, to simplify the discussions we consider that the interest rate is zero.

Let us assume that $\mathcal{X} \subset L^{p}$ is a closed convex cone containing $\mathbb{R}$ (the set of real numbers), representing all viable pay-offs, i.e. for every $X \in \mathcal{X}$ there is a price associated with $X$.

Definitio 1 A continuous mapping $\pi: \mathcal{X} \rightarrow \mathbb{R}$ is a sub-linear pricing rule if

(i) $\pi(X+k)=\pi(X)+k, \forall X \in \mathcal{X}, \forall k \in \mathbb{R}$;

(ii) $\pi(\lambda X)=\lambda \pi(X), \forall X \in \mathcal{X}, \forall \lambda>0$;

(iii) $\pi(X+Y) \leq \pi(X)+\pi(Y), \forall X, Y \in \mathcal{X}$;

(iv) $\pi(X) \leq \pi(Y), \forall X, Y \in L^{p}$ and $X \leq Y$.

Remark 1 The pricing rule $\pi$ can be for example considered the super-replication price, when $\mathcal{X}$ consists of all random variables like $X$ such that there exists a viable self-financin process which can super-hedge $X$.

Definitio 2 A continuous mapping $\rho: L^{p} \rightarrow \mathbb{R}$ is a coherent risk measure if

(1) $\rho(X+k)=\rho(X)-k$ for every $X \in L^{p}$ and $k \in \mathbb{R}$;

(2) $\rho(\lambda X)=\lambda \rho(X)$ for every $X \in L^{p}$ and $\lambda>0$;

(3) $\rho(X+Y) \leq \rho(X)+\rho(Y)$ for every $X, Y \in L^{p}$;

(4) $\rho(X) \leq \rho(Y)$ for every $X, Y \in L^{p}$ and $X \geq Y$.

A particularly interesting example is the Conditional Value at Risk (CVaR) of Rockafellar et al. [18]. Let

$$
\Delta_{\rho}:=\left\{Z \in L^{q} \mid-\mathbb{E}(X Z) \leq \rho(X), \forall X \in L^{p}\right\} .
$$

The set $\Delta_{\rho}$ is obviously convex. Bearing in mind the Representation Theorem 2.4.9 in Zalinescu [19] for $p \neq \infty$, and using a proof similar to that of the Representation Theorem of a risk measure, from what is stated in Rockafellar et al. [18], it can be seen that $\Delta_{\rho}$ is $\sigma\left(L^{q}, L^{p}\right)$-compact, and

$$
\rho(X)=\max _{Z \in \Delta_{\rho}} \mathbb{E}[-Z X], \quad \forall X \in L^{p} .
$$

Furthermore, by (1) and (4) one can see that

$$
\Delta_{\rho} \subset\left\{Z \in L_{+}^{q} \mid \mathbb{E}(Z)=1\right\} .
$$

By means of the Hahn-Banach Separation Theorem, one can easily prove that if $\Delta_{\rho} \subset L^{q}$ is convex, $\sigma\left(L^{q}, L^{p}\right)$-compact and $\Delta_{\rho}$ satisfie (2.3), then there exists a unique continuous $\rho$ satisfying (1), (2), (3) and (4) such that (2.2) holds.

For $p=\infty$, in order to have the same representation, $\rho$ needs to have the Fatou property introduced in Delbaen [11]. We say that $\rho$ has the Fatou property if for any bounded 
sequence $\left\{X_{n}\right\}_{n=1}^{\infty} \subseteq L^{\infty}$ converging in probability to $X$ we have that $\rho(X) \leq \rho\left(X_{n}\right)$. For coherent risk measures this is equivalent to the continuity from above i.e. for every sequence $\left\{X_{n}\right\}_{n=1}^{\infty}$ in $L^{\infty}$ such that $X_{n} \downarrow X$ we have that $\rho\left(X_{n}\right) \rightarrow \rho(X)$ (see Delbaen [11]). With this assumption $\Delta_{\rho}$ is a subset of $L^{1}$, but not in general $\sigma\left(L^{1}, L^{\infty}\right)$-compact. In the sequel for $p=\infty$ we also add the assumption that $\Delta_{\rho}$ is $\sigma\left(L^{1}, L^{\infty}\right)$-compact, which with the aid of the Dunford-Pettis Theorem means that $\Delta_{\rho}$ is Uniformly Integrable. It is worth mentioning that the $\sigma\left(L^{1}, L^{\infty}\right)$-compactness is equivalent to the so-called Lebesgue property of $\rho$ define in Jouini et al. [17]. A coherent risk measure $\rho$ satisfie the Lebesgue property if for any bounded sequence $\left\{X_{n}\right\}_{n=1}^{\infty} \subseteq L^{\infty}$ converging in probability to $X$ we have that $\rho\left(X_{n}\right) \rightarrow \rho(X)$. For coherent risk measures this property is also equivalent to the continuity from below i.e. if $X_{n} \uparrow X$ then $\rho\left(X_{n}\right) \rightarrow \rho(X)$. For further discussions see for example Föllmer and Schied [14] Proposition 4.21. It is also important to know that most common law invariant coherent (convex in general) risk measures display this property. For instance, for the coherent risk measure $\mathrm{CVaR}_{\alpha}$ (where $\alpha \in(0,1)$ is a confidenc level) we know that $\Delta_{\mathrm{CVaR}_{\alpha}}=\left\{f: \Omega \rightarrow \mathbb{R} \mid 0 \leq f \leq \frac{1}{\alpha}, \mathbb{E}[f]=1\right\}$ is Uniformly Integrable (and hence $\sigma\left(L^{1}, L^{\infty}\right)$-compact). It is shown in Delbaen [12] that a law invariant coherent (convex in general) risk measure on $L^{\infty}$ is continuous from below if and only if its extension to $L^{1}$ takes finit value for some position which is unbounded from below. This is important to know because we will see that any coherent risk measure define on $L^{1}$ is incompatible with pricing rules induced by unbounded stochastic discount factors (like one given by the Black-Scholes model).

\section{Compatibility and Good Deals}

This section will be devoted to introduce the notion of compatibility between a coherent risk measure and a sub-linear pricing rule and its relation with Good Deals.

Definitio 3 Let $\pi$ be a sub-linear pricing rule and $\rho$ a coherent risk measure. We say $\pi$ and $\rho$ are compatible if there is no sequence $\left(X_{n}\right)_{n=1}^{\infty} \subset \mathcal{X}$ such that the following conditions simultaneously hold

$$
\begin{aligned}
& \pi\left(X_{n}\right) \leq 0, \quad \forall n \in \mathbb{N} \\
& \lim _{n \rightarrow \infty} \rho\left(X_{n}\right)=-\infty .
\end{aligned}
$$

We say $\pi$ and $\rho$ are incompatible if they are not compatible.

As one can see if $\pi$ and $\rho$ are incompatible, then every manager who uses $\rho$ to assess the risk can make the risk as negative as he/she wishes, which does not make any economical sense. For further discussion we refer the reader to Balbás et al. [2].

Now we give our definition of a Good Deal inspired by definitions in Černý and Hodges [7] and Cherny [9].

Definitio 4 A Good Deal is a position $X \in \mathcal{X}$ such that $\pi(X) \leq 0$ and $\rho(X)<0$. If there is no Good Deal then we say that the No Good Deal assumption holds.

Theorem 3.1 Let $\rho$ be a coherent risk measure and $\pi$ a sub-linear pricing rule. Let

$$
\mathcal{R}:=\left\{Z \in L_{+}^{q} \mid \mathbb{E}[Z]=1, \pi(X)-\mathbb{E}(X Z) \geq 0, \forall X \in \mathcal{X}\right\} .
$$


The No Good Deal assumption holds if and only if

$$
\Delta_{\rho} \cap \mathcal{R} \neq \emptyset .
$$

Proof This is easily concluded by using Theorem 3.4 in Cherny [9].

Here we present an example of a Good Deal, illustrating how these pathological positions could appear in a market.

Example Consider a random variable $Y \in L^{1} \backslash L^{2}$. Without lose of generality one can consider that $Y$ is bounded above by a positive number $M$ (otherwise one can pick either $-|Y| 1_{\{Y<0\}}+M$ or $-|Y| 1_{\{Y \geq 0\}}+M$ in lieu of $Y$ ). Let $\rho$ be any law invariant risk measure on $L^{\infty}$. Since $\rho$ is law invariant, it is finit on $L^{1}$ (see Remark 2) which implies that $\rho(Y)<\infty$. Let $X_{n}=Y 1_{\{Y \geq-n\}}+\rho(Y)$ and note that $\rho\left(X_{n}\right) \uparrow 0$. Let $\mathcal{X}=L^{\infty}$ and defin $\pi(X)=\mathbb{E}\left(\frac{1}{\|Y\|_{L^{1}}}|Y| X\right)$. Considering the above notations, we have

$$
\begin{aligned}
\pi\left(X_{n}\right) & =\pi\left(Y 1_{\{Y \geq-n\}}+\rho(Y)\right) \\
& =\pi\left(Y 1_{\{Y \geq-n\}}-M\right)+M+\rho(Y) \\
& =\pi\left(-\left|Y 1_{\{Y \geq-n\}}-M\right|\right)+M+\rho(Y) \\
& \leq \frac{1}{\|Y\|_{L^{1}}} \mathbb{E}\left(-Y^{2} 1_{\{Y \geq-n\}}\right)+2 M+\rho(Y) \stackrel{n \rightarrow \infty}{\longrightarrow}-\infty,
\end{aligned}
$$

where in the last line we used the fact that $Y \notin L^{2}$. One can see that for $n$ large enough we have a position $X_{n}$ such that $\rho\left(X_{n}\right) \leq 0$ whereas $\pi\left(X_{n}\right)<0$. By definition $X_{n}$ is a Good Deal. In addition, according to definition of incompatibility, $\rho$ and $\pi$ are incompatible (use $X_{n}$ in the definition).

\subsection{A hedging problem}

Here we consider a more practical discussion when we want to hedge a financia position $g$ with all possible choices we can make subject to a given budget constraint over a set $\mathcal{X}$. This problem will help us to better discover the relation between the concepts of incompatibility and Good Deals.

Let us consider the following problem

$$
\left\{\begin{array}{l}
\min \rho(X-g)+c \\
\pi(X) \leq c \\
X \in \mathcal{X}, \quad c \in \mathbb{R} .
\end{array}\right.
$$

This problem has been studied in Balbás et al. $[3,2,4]$. The dual of problem (3.4) is found in Balbás et al. [3] as

$$
\left\{\begin{array}{l}
\max \mathbb{E}[g Z] \\
Z \in \mathcal{R} \cap \Delta_{\rho} .
\end{array}\right.
$$

Following the discussions in Balbás et al. $[3,2,4]$ we have the following theorem

Theorem 3.2 The following statements are equivalent

1. $\pi$ and $\rho$ are compatible.

2. $\mathcal{R} \cap \Delta_{\rho} \neq \emptyset$.

3. Problem (3.4) is bounded. 


\section{Problem (3.5) has a feasible solution.}

5. There is no duality gap between (3.4) and (3.5).

As one can see (3.5) has a solution if and only if $\Delta_{\rho} \cap \mathcal{R} \neq \emptyset$, which obviously reminds us of Theorem 3.1. Now we add the following statements to Theorem 3.2

\section{Theorem 3.3 The statements of Theorem 3.2 are equivalent to the following statements}

1. The No Good Deal assumption holds.

2. $\rho+\pi \geq 0$.

Proof With the aid of Theorem 3.1 it is clear that statement 2 of Theorem 3.2 is equivalent to statement 1 of Theorem 3.3. Now we prove that statements 1 and 2 of Theorem 3.3 are equivalent. From Definition 4, it is obvious that the No Good Deal assumption holds iff for all $X$ in $\mathcal{X}, \pi(X) \leq 0$ implies $\rho(X) \geq 0$. Therefore, since $\pi(X-\pi(X))=0$, we must have $\rho(X-\pi(X)) \geq 0$. Since $\rho$ is translation invariant, we conclude that $\rho(X)+\pi(X) \geq 0$, showing that 1 implies 2 . Now we prove the other implication. To this end, let us suppose that there exists a Good Deal $X \in \mathcal{X}$. By Definition 4, there exists $X \in \mathcal{X}$ such that $\rho(X)<0$ and $\pi(X) \leq 0$ which imply $\rho(X)+\pi(X)<0$.

In the following remark we show that Good Deals are not rare positions.

Remark 2 Suppose that $p \neq 1$. Let $\rho$ be a law invariant coherent risk measure on $L^{p}$ i.e. $\rho(X)=\rho(Y)$ for any two random variables $X, Y$ with identical distributions. It has recently been proven in Filipovic and Svindland [13] that every law invariant coherent risk measure on $L^{\infty}$ can canonically be extended to $L^{1}$. Let us for a moment denote this extension by $\tilde{\rho}$. According to previous discussions, $\Delta_{\tilde{\rho}}$ is a $\sigma\left(L^{\infty}, L^{1}\right)$-closed convex set of $L^{\infty}$. Since $L^{p} \subseteq L^{1}, \Delta_{\rho}$ is also $\sigma\left(L^{q}, L^{p}\right)$-closed convex set of $L^{q}$. This implies that $\tilde{\rho}$ restricted to $L^{p}$ can be represented as $\tilde{\rho}(X)=\sup _{Z \in \Delta_{\tilde{\rho}}} \mathbb{E}[-X Z]$ which implies, since $\Delta_{\tilde{\rho}}$ is $\sigma\left(L^{q}, L^{p}\right)$ closed, that $\Delta_{\rho}=\Delta_{\tilde{\rho}}$. As a consequence one can see that $\Delta_{\rho}$ is a subset of $L^{\infty}$. Now, according to Theorem 3.1, this shows that any law invariant coherent risk measure can produce Good Deals with any pricing rule with unbounded stochastic discount factors. A particular interesting example is the risk measure CVaR (which is law invariant) and the pricing rule given by the Black-Scholes model (which is unbounded).

\section{Risk modificatio}

Discussions in the Remark 2 show that compatibility may fail in very important cases. Now it is natural to analyze whether modification of a risk measure allows us to recover this situation.

Definitio 5 Let $\pi$ be a sub-linear pricing rule and $\rho$ a coherent risk measure. A minimal compatible modification denoted by $\rho_{m}$, is a coherent risk measure such that:

(a) $\pi$ and $\rho_{m}$ are compatible, and $\rho \leq \rho_{m}$;

(b) $\rho_{m}$ is minimal, i.e. for any risk measure $\tilde{\rho}$ such that $\pi$ and $\tilde{\rho}$ are compatible and $\rho \leq$ $\tilde{\rho} \leq \rho_{m}$, we have that $\tilde{\rho}=\rho_{m}$.

Note that the minimal compatible modificatio is not necessarily unique.

To study the existence of minimal compatible modificatio we need the following notation. For a given $Z \notin \Delta_{\rho}$ let

$$
C(Z):=\operatorname{co}\left(\{Z\} \cup \Delta_{\rho}\right) .
$$


where by co we mean the convex hull. It is easy to see that since $\Delta_{\rho}$ is $\sigma\left(L^{p}, L^{q}\right)$-compact then $C(Z)$ is $\sigma\left(L^{p}, L^{q}\right)$-closed. Defin $\preceq$ for two members $Z_{1}, Z_{2} \notin \Delta_{\rho}$ :

$$
Z_{1} \preceq Z_{2} \Leftrightarrow C\left(Z_{1}\right) \subseteq C\left(Z_{2}\right) .
$$

Easily one can give the following equivalent definition for $\preceq$ :

$$
Z_{1} \preceq Z_{2} \Leftrightarrow Z_{1} \in C\left(Z_{2}\right)
$$

This relation shows that $\preceq$ is a transitive relation. In the following theorem we see that if $\mathcal{R} \cap \Delta_{\rho}=\emptyset$, at least one minimal member of this partial ordering exists.

Theorem 4.1 Suppose that $\mathcal{R} \cap \Delta_{\rho}=\emptyset$. Then there exists a minimal point $Z \in(\mathcal{R}, \preceq)$.

Before proving the theorem we need to prove the following lemma

Lemma 1 Let $\left\{Z_{n}\right\}_{n=1}^{\infty}$ be a sequence in $\mathcal{R}$ such that $Z_{1} \succeq Z_{2} \succeq Z_{3} \succeq \ldots$ and $Z_{n} \rightarrow Z$ in $\sigma\left(L^{q}, L^{p}\right)$. Then

$$
\cap_{n \in \mathbb{N}} C\left(Z_{n}\right)=C(Z) .
$$

Proof Fix an arbitrary integer number $N \in \mathbb{N}$. By our assumption we have $Z_{n} \preceq Z_{N}, \forall n \geq N$. Hence, $Z_{n} \in C\left(Z_{N}\right), \forall n \geq N$. Since $C\left(Z_{N}\right)$ is closed and $N$ is arbitrarily chosen, we deduce that $Z \in C\left(Z_{N}\right)$. That gives $\forall N \geq 1, C(Z) \subseteq C\left(Z_{N}\right)$ which yields $C(Z) \subseteq \cap_{n \in \mathbb{N}} C\left(Z_{n}\right)$.

For the other implication let $\tilde{Z}$ be a member of $\cap_{n \in \mathbb{N}} C\left(Z_{n}\right)$. For any $n \in \mathbb{N}$, by definition of $C\left(Z_{n}\right)$ there exists $\lambda_{n} \in[0,1]$ and $Z_{n}^{*} \in \Delta_{\rho}$ such that

$$
\tilde{Z}=\left(1-\lambda_{n}\right) Z_{n}+\lambda_{n} Z_{n}^{*} \text {. }
$$

Since $\Delta_{\rho}$ is $\sigma\left(L^{q}, L^{p}\right)$-compact and $[0,1]$ is bounded, one can extract convergent subsequences from $Z_{n}^{*}$ and $\lambda_{n}$ converging to $Z^{*} \in \Delta_{\rho}$ and $\lambda \in[0,1]$ respectively. In the limit we have

$$
\tilde{Z}=(1-\lambda) Z+\lambda Z^{*},
$$

which means that $\tilde{Z}$ belongs to the convex hull of $Z$ and $\Delta_{\rho}$. By definition of $C(Z)$ this gives that $\tilde{Z} \in C(Z)$ and therefore the proof of the lemma is complete.

Proof of Theorem 4.1 Fix a member $\bar{Z}$ in $\mathcal{R}$ and let

$$
\mathcal{A}=\{Z \in C(\bar{Z}) \cap \mathcal{R} \mid Z \preceq \bar{Z}\} .
$$

We show that $(\mathcal{A}, \succeq)$ satisfie the conditions of Zorn's Lemma. Since $\bar{Z} \in C(\bar{Z})$, the set $\mathcal{A}$ is obviously nonempty. On the other hand let $\left\{Z_{n}\right\}_{n=1}^{\infty}$ be a chain in $\mathcal{A}$ i.e. $Z_{1} \succeq Z_{2} \succeq \ldots$. Since $\mathcal{A}$ is $\sigma\left(L^{q}, L^{p}\right)$-compact, there exists a subsequence $\left\{Z_{n_{k}}\right\}_{k=1}^{\infty}$ such that $Z_{n_{k}} \rightarrow Z$ in $\sigma\left(L^{q}, L^{p}\right)$, for some $Z \in \mathcal{A}$. By applying Lemma 1 and using the fact that $C\left(Z_{1}\right) \supseteq$ $C\left(Z_{2}\right) \supseteq \ldots$ we have that $\cap_{i \in \mathbb{N}} C\left(Z_{i}\right)=C(Z)$. This means that $Z$ is a supremal point of the chain. By applying Zorn's Lemma, there exists a $\succeq$-supremal, hence $\preceq$-minimal point $Z \in \mathcal{A}$.

Now we claim that $Z$ is a minimal point for $\mathcal{R}$. Suppose there exists $\tilde{Z}$ in $\mathcal{R}$ such that $\tilde{Z} \preceq Z$. Since $\tilde{Z} \preceq Z \preceq \bar{Z}$ and since $\preceq$ is transitive [see (4.3)] we have that $\tilde{Z} \in C(\bar{Z})$ which by definition gives $\tilde{Z} \in \mathcal{A}$. Since $Z$ is a minimal point for $(\mathcal{A}, \preceq)$ consequently $Z=\tilde{Z}$ which implies that $Z$ is minimal for $(\mathcal{R}, \preceq)$.

Now the proof of the following theorem is straightforward and we leave it to the reader 
Theorem 4.2 Suppose that No Good Deal assumption does not hold. The risk measure $\rho_{m}$ is a minimal compatible modification of $\rho$ if and only if

$$
\Delta_{\rho_{m}}=C(Z)
$$

for some minimal member $Z$ in $(\mathcal{R}, \preceq)$.

The following corollary gives a perfect geometrical description of a minimal compatible extension of a coherent risk measure in terms of the ordered set $(\mathcal{R}, \preceq)$. This corollary modifie Theorem 2 in Balbás and Balbás [1].

Corollary 1 (Minimal Modification) Suppose that No Good Deal assumption does not hold and $\rho_{m}$ is a minimal modification of $\rho$. Then

$$
\rho_{m}(X)=\max \{\rho(X),-\mathbb{E}(Z X)\}
$$

for some minimal point $Z$ in $(\mathcal{R}, \preceq)$.

Proof This is a direct implication of Theorems 4.1 and 4.2.

\section{Modificatio rules}

In the following discussions we propose two major methods for findin a minimal compatible modificatio $\rho_{m}$ of $\rho$. The firs method relies on minimizing a third function $\phi$, which is interpreted as a spread criteria. This new measure $\phi$ concerns the fundamentals of the $\rho$-user. For instance, we will see that, by supposing $\phi(\cdot)=\|\cdot\|_{L^{1}}, \Delta_{\rho}$ does not spread out very far in terms of the $L^{1}$-norm.

As for the second proposed way of modifying the risk measure, our method is based on the No Better Choice (NBC) pricing method introduced in Cherny [9] for the so-called Global/Local Efficiency Ratio. The Global/Local Efficiency Ratio is a performance ratio which takes the market fundamentals as well as the risk user desires into account.

\subsection{Minimal risk spread}

Let us start with the following definition

Definitio 6 A function $\phi: L^{q} \rightarrow \mathbb{R}$ is a spread criteria if

$(\phi 1) \phi$ is positive and convex.

( $\phi 2)$ The function $\left(Z, Z_{1}\right) \mapsto \phi\left(Z-Z_{1}\right)$ attained its minimum at a point $\left(Z_{\text {min }}, Z^{*}\right) \in$ $\mathcal{R} \times \Delta_{\rho}$.

( $\phi 3)$ The equality $\phi(Z)=0$ holds if and only if $Z=0$.

The following theorem enables us to fin a minimal compatible modificatio of a coherent risk measure $\rho$ based on a spread criteria $\phi$.

Theorem 5.1 Suppose that the No Good Deal assumption does not hold. Then, in the above notation $Z_{\text {min }}$ is a minimal point for $(\mathcal{R}, \preceq)$.

Proof Since No Good Deal assumption does not hold, by Theorem 3.3 we know that $\mathcal{R} \cap$ $\Delta_{\rho}=\emptyset$. To prove the theorem's statement we suppose, to the contrary, that $Z_{\min }$ is not minimal. Then there exists $\tilde{Z} \in \mathcal{R}$ such that $\tilde{Z} \in C\left(Z_{\text {min }}\right)$ and $\tilde{Z} \neq Z_{\text {min }}$. Since $Z_{\text {min }} \neq$ $\tilde{Z} \in C\left(Z_{\text {min }}\right)$, by definition there exists $\lambda \in(0,1]$ and $Z_{1} \in \Delta_{\rho}$ such that

$$
\tilde{Z}=(1-\lambda) Z_{\min }+\lambda Z_{1} \text {. }
$$


Let $Z_{2}=(1-\lambda) Z^{*}+\lambda Z_{1}$. By convexity of $\Delta_{\rho}$ we know that $Z_{2} \in \Delta_{\rho}$. Given assumptions $(\phi 1),(\phi 3)$ we have

$$
\begin{aligned}
\phi\left(\tilde{Z}-Z_{2}\right) & =\phi\left((1-\lambda) Z_{\text {min }}+\lambda Z_{1}-\left((1-\lambda) Z^{*}+\lambda Z_{1}\right)\right) \\
& =\phi\left((1-\lambda)\left(Z_{\text {min }}-Z^{*}\right)\right) \\
& \leq(1-\lambda) \phi\left(Z_{\min }-Z^{*}\right) .
\end{aligned}
$$

Since $0 \leq 1-\lambda<1$, by definition of $Z_{\min }$ we have that $\phi\left(Z_{\min }-Z^{*}\right)=0$. By condition $(\phi 3)$ we get that $Z_{\min }=Z^{*}$ which contradicts our Good Deal assumption.

\subsubsection{Compatible conditional value at risk (CCVaR)}

In this part we are going to use the theory that have been developed in the last section by implementing $\phi(X)=\mathbb{E}[|X|]$ and $\rho=\mathrm{CVaR}_{\alpha}$ for some confidenc level $\alpha \in(0,1)$. Interestingly, we will see that in order to fin the CCVaR, we will have to fin a stochastic discount factor with the least European call option price with strike price $\frac{1}{\alpha}$.

Lemma 2 For a given $g \in L_{+}^{1}$ with $\mathbb{E}[g]=1$, the $L^{1}$-distance between $g$ and $\Delta_{\mathrm{CVaR}_{\alpha}}$ is equal to $2 \mathbb{E}\left[\left(g-\frac{1}{\alpha}\right)^{+}\right]$i.e.

$$
\min _{Z \in \Delta_{\mathrm{CVaR} \alpha}} \mathbb{E}[|g-Z|]=2 \mathbb{E}\left[\left(g-\frac{1}{\alpha}\right)^{+}\right] .
$$

Furthermore, the minimum is attained only in points $Z^{*}$ given as

$$
Z^{*}=\frac{1}{\alpha} 1_{\left\{g \geq \frac{1}{\alpha}\right\}}+(g+h) 1_{\left\{g<\frac{1}{\alpha}\right\}},
$$

where $h$ is a non-negative function for which $(g+h) 1_{\left\{g<\frac{1}{\alpha}\right\}} \leq \frac{1}{\alpha}$ and

$$
\mathbb{E}\left[1_{\left\{g<\frac{1}{\alpha}\right\}} h\right]=\mathbb{E}\left[\left(g-\frac{1}{\alpha}\right)^{+}\right] .
$$

Proof First recall that

$$
\Delta_{\mathrm{CVaR}_{\alpha}}=\left\{f \in L^{1} \mid 0 \leq f \leq \frac{1}{\alpha}, \mathbb{E}[f]=1\right\} .
$$

Let $Z \in \Delta_{\mathrm{CVaR}_{\alpha}}$ and defin

$$
\begin{aligned}
& Z_{1}:=(Z-g) 1_{Z \geq g}, \\
& Z_{2}:=(g-Z) 1_{\left\{g \geq Z, g<\frac{1}{\alpha}\right\}}, \\
& Z_{3}:=\min (Z, g), \\
& Z_{4}:=\left(\frac{1}{\alpha}-Z\right) 1_{g \geq \frac{1}{\alpha}}, \\
& Z_{5}:=\left(g-\frac{1}{\alpha}\right) 1_{g \geq \frac{1}{\alpha}} .
\end{aligned}
$$

It is clear that

$$
\begin{aligned}
& Z_{1}+Z_{3}=Z, \\
& g=Z_{2}+Z_{3}+Z_{4}+Z_{5} .
\end{aligned}
$$


Therefore, denoting by $\int$ the expectation with respect to $\mathbb{P}$,

$$
\begin{aligned}
& \int Z_{1}+\int Z_{3}=1, \\
& 1=\int Z_{2}+\int Z_{3}+\int Z_{4}+\int Z_{5} .
\end{aligned}
$$

On the other hand, since $Z_{2}$ and $Z_{4}$ are non-negative we have

$$
2 \int Z_{2}+\int Z_{4} \geq 0
$$

Combining (5.5) with (5.3) and (5.4)

$$
\int Z_{1}+\int Z_{2} \geq \int Z_{5}
$$

Adding one more $\int Z_{5}$ to the both sides of the last inequality, we obtain

$$
\int Z_{1}+\int Z_{2}+\int Z_{5} \geq 2 \int Z_{5}
$$

Having this, one can see that

$$
\begin{aligned}
\int|Z-g| & =\int Z_{1}+\int Z_{2}+\int_{g \geq \frac{1}{\alpha}}(g-Z) \\
& \geq \int Z_{1}+\int Z_{2}+\int Z_{5} \\
& \geq 2 \int Z_{5}=2 \int\left(g-\frac{1}{\alpha}\right)^{+} .
\end{aligned}
$$

Therefore, $2 \int\left(g-\frac{1}{\alpha}\right)^{+}$is smaller than $\int|Z-g|$ for all $Z$.

Now we take three steps to conclude the proof: First, we show at least one $Z^{*}$ exists. Second, we show every $Z^{*}$ introduced in (5.1) is a minimal point. Third, we prove every minimal point has the same structure as in (5.1).

Step 1. We show that there exists a function $h$ which satisfie the conditions in Lemma 2 and can be put into (5.1).

Observe that since $0>1-\frac{1}{\alpha}=\int\left(g-\frac{1}{\alpha}\right)=\int_{g \geq \frac{1}{\alpha}}\left(g-\frac{1}{\alpha}\right)+\int_{g<\frac{1}{\alpha}}\left(g-\frac{1}{\alpha}\right)$ we have $\int_{g \geq \frac{1}{\alpha}}\left(g-\frac{1}{\alpha}\right)<\int_{g<\frac{1}{\alpha}}\left(\frac{1}{\alpha}-g\right)$. Let $\lambda:=\frac{\int_{g \geq \frac{1}{\alpha}}\left(g-\frac{1}{\alpha}\right)}{\int_{g<\frac{1}{\alpha}}\left(\frac{1}{\alpha}-g\right)}$, and note that $\lambda<1$. By definin $h:=\lambda\left(\frac{1}{\alpha}-g\right) 1_{g<\frac{1}{\alpha}}$, obviously $h$ fulfill the conditions of Lemma 2 .

Step 2. Suppose that $h$ is a non-negative function for which $(g+h) 1_{\left\{g<\frac{1}{\alpha}\right\}} \leq \frac{1}{\alpha}$ and (5.2) holds. Defin

$$
Z^{*}:=\frac{1}{\alpha} 1_{g \geq \frac{1}{\alpha}}+(g+h) 1_{g<\frac{1}{\alpha}}
$$


First we show that $Z^{*} \in \Delta_{\mathrm{CVaR}_{\alpha}}$. By construction it is clear that $0 \leq Z^{*} \leq \frac{1}{\alpha}$. On the other hand by (5.2) we have that

$$
\begin{aligned}
\int_{\Omega} Z^{*} & =\int_{g \geq \frac{1}{\alpha}} \frac{1}{\alpha}+\int_{g<\frac{1}{\alpha}} g+\int_{g<\frac{1}{\alpha}} h \\
& =\int_{g \geq \frac{1}{\alpha}} \frac{1}{\alpha}+\int_{g<\frac{1}{\alpha}} g+\int_{\Omega}\left(g-\frac{1}{\alpha}\right)^{+} \\
& =\int_{g \geq \frac{1}{\alpha}} \frac{1}{\alpha}+\int_{g<\frac{1}{\alpha}} g+\int_{g \geq \frac{1}{\alpha}}\left(g-\frac{1}{\alpha}\right) \\
& =\int_{\Omega} g=1 .
\end{aligned}
$$

Now we show that $Z_{2}^{*}=Z_{4}^{*}=0$. It is easy to see that $Z_{4}^{*}=0$. As for $Z_{2}^{*}=0$, just observe that by definition of $Z^{*},\left\{g<\frac{1}{\alpha}, g \geq Z^{*}\right\}=\{h=0\}$, and therefore

$$
Z_{2}^{*}=\left(g-Z^{*}\right) 1_{\left\{g \geq Z^{*}, g<\frac{1}{\alpha}\right\}}=-h 1_{\left\{g \geq Z^{*}, g<\frac{1}{\alpha}\right\}}=-h_{\{h=0\}}=0 .
$$

On the other hand, it is also clear that $\left(g-Z^{*}\right) 1_{\left\{g \geq \frac{1}{\alpha}\right\}}=0$. Given this, since $Z_{2}^{*}=Z_{4}^{*}=0$, we have equalities in (5.7) and (5.8), which implies that $Z^{*}$ is a minimal point.

Step 3. From Steps 1,2 it is clear that the minimum is

$$
2 \int\left(g-\frac{1}{\alpha}\right)^{+}
$$

This, along with (5.7) and (5.8), shows that for any minimal point $Z^{*} \in \Delta_{\rho}$ we must have $Z_{2}^{*}=Z_{4}^{*}=0$. The equality $Z_{4}^{*}=0$ implies that

$$
Z^{*} 1_{g \geq \frac{1}{\alpha}}=\frac{1}{\alpha}
$$

This is the firs part of (5.1).

Let $h:=\left(Z^{*}-g\right) 1_{\left\{g<\frac{1}{\alpha}\right\}}$. By construction, $Z^{*} 1_{\left\{g<\frac{1}{\alpha}\right\}}=(h+g) 1_{\left\{g<\frac{1}{\alpha}\right\}}$, which is the second part of (5.1).

Now we must show that $h$ is non-negative, $(g+h) 1_{\left\{g<\frac{1}{\alpha}\right\}} \leq \frac{1}{\alpha}$ and that (5.2) holds. From $0=Z_{4}^{*}=\left(g-Z^{*}\right) 1_{\left\{g \geq Z^{*}, g<\frac{1}{\alpha}\right\}}$ it turns out that $g$ cannot be larger than $Z^{*}$ on $\left\{g<\frac{1}{\alpha}\right\}$. This implies that the function $h=\left(Z^{*}-g\right) 1_{\left\{g<\frac{1}{\alpha}\right\}}$ is non-negative. Since $Z^{*} \leq \frac{1}{\alpha}$, it is also clear that $(g+h) 1_{\left\{g<\frac{1}{\alpha}\right\}} \leq \frac{1}{\alpha}$. 
By assumption that $Z^{*}$ is minimal, definition of $h$ and (5.9) we have that

$$
\begin{aligned}
2 \int\left(g-\frac{1}{\alpha}\right)^{+} & =\int\left|g-Z^{*}\right| \\
& =\int_{g \geq \frac{1}{\alpha}}\left(g-\frac{1}{\alpha}\right)+\int_{g<\frac{1}{\alpha}} h \\
& =\int\left(g-\frac{1}{\alpha}\right)^{+}+\int_{g<\frac{1}{\alpha}} h,
\end{aligned}
$$

which shows that (5.2) hold and the proof is complete.

From Theorem 5.1 and Lemma 2 we deduce the following theorem:

Theorem 5.2 Let SDF be the set of all Stochastic Discount Factors (e.g. EMM in an incomplete market). Suppose that the minimum of $2 \mathbb{E}\left[\left(\cdot-\frac{1}{\alpha}\right)^{+}\right]$over $S D F$ is attained at $g^{*} \in S D F$. Then $g^{*}$ is a minimal point of (SDF, $\left.\preceq\right)$.

Remark 3 Interestingly one can see that findin the minimal extension for CVaR is equivalent to findin a stochastic discount factor with the least European call option price with strike $\frac{1}{\alpha}$.

Remark 4 In an incomplete market, there is more than one equivalent martingale measure. Among many choices, the right pick is always an important question. For instance, the minimal martingale measure provided by the Föllmer Schweizer decomposition, the one which is the nearest in $L^{q}$-norm to the historical measure $\mathbb{P}$, or the one which has the least entropy could be named among many (see Chan [8]). Here, we can add another to this list, which concerns the existence of Good Deals.

\subsection{Global risk and performance maximization}

In this section we propose the second way of modifying a risk measure which will be carried out via studying the following coherent risk measure:

$$
X \mapsto G R(X):=\max \{\rho(X), \pi(-X)\} .
$$

This is what we call the Global Risk measure. Indeed, the Global Risk does not only assess the trader's risk, but also the market response to going short on $X$, which could be interpreted as the market risk. As usual in the literature of coherent risk measure, in the sequel, we will denote the function $-\rho$ by $u$, and we will call it the monetary utility associated with $\rho$.

For our discussions in this section we need the following assumption on $\mathcal{R}$ :

$$
\mathcal{R} \text { is } \sigma\left(L^{q}, L^{p}\right) \text {-compact. }
$$

Now we start to study the efficien y ratio $\frac{u(X)}{G R(X)}$ in order to propose another way of findin a minimal compatible modificatio of risk measure $\rho$. We have the following definition

Definitio 7 For a couple $(\pi, \rho)$ the Global/Local performance ratio $G L$ is define as follows:

$$
G L(X)=\left\{\begin{array}{cl}
+\infty & \text { if } G R(X)<0 \\
\frac{u(X)}{G R(X)} & \text { if } G R(X) \geq 0 \text { and } u(X)>0, \\
0 & \text { if } G R(X) \geq 0 \text { and } u(X) \leq 0
\end{array}\right.
$$


when $\frac{\text { positive }}{0}=+\infty$.

It is easy to show that

$$
G L(X)=\left\{\begin{array}{cl}
+\infty & \text { if } u(X)>0 \text { and } \pi(-X) \leq 0 \\
\frac{u(X)}{\pi(-X)} & \text { if } u(X)>0 \text { and } \pi(-X)>0 \\
0 & \text { if } u(X) \leq 0
\end{array}\right.
$$

This is a measure to see how much it is worth to keep $X$.

Now let us suppose that the No Good Deal assumption holds. Let $X$ be a financia position such that $\pi(X) \leq 0$. It is clear since $\mathcal{R} \cap \Delta_{\rho} \neq \emptyset$ then $u(X) \leq 0$, and by (5.12) we have $G L(X)=0$. However, in the opposite case, when the No Good Deal assumption does not hold, i.e. $\mathcal{R} \cap \Delta_{\rho}=\emptyset$, we always have $\sup _{\pi(X) \leq 0} G L(X)>0$. This number shows how far a market is from the No Good Deal assumption. We have the following proposition

Proposition 1 The No Good Deal assumption holds if and only if $G L(X)=0$ for all $X$ in $\{\pi \leq 0\}$.

Here we lead the discussion to the No Better Choice pricing rule associated with the performance ratio $G L$ define by Cherny [9].

Definitio 8 For any financia position $g$ the NBC price of $g$ is a real number $x$ such that

$$
\sup _{\{X+h(g-x) \mid \pi(X) \leq 0, h \in \mathbb{R}\}} G L(X+h(g-x))=\sup _{\{X \mid \pi(X) \leq 0\}} G L(X) .
$$

Actually it is the cost for $g$ in which the maximum efficien y ratio does not increase by adding the new product $g$.

We denote the supremum in $(5.13)$ by $R^{*}$, i.e.

$$
R^{*}=\sup _{\{X \mid \pi(X) \leq 0\}} G L(X) .
$$

In Cherny [9] it is shown that

$$
R^{*}=\inf \left\{R \geq 0 \mid\left(\frac{1}{1+R} \Delta_{\rho}+\frac{R}{1+R} \overline{\operatorname{co}}\left(\Delta_{\rho} \cup \mathcal{R}\right)\right) \cap \mathcal{R} \neq \emptyset\right\} .
$$

Since $\Delta_{\rho}$ and $\mathcal{R}$ are $\sigma\left(L^{q}, L^{p}\right)$-compact and both $\Delta_{\rho}$ and $\mathcal{R}$ are convex it is clear that

$$
\overline{c o}\left(\Delta_{\rho} \cup \mathcal{R}\right)=\operatorname{co}\left(\Delta_{\rho} \cup \mathcal{R}\right) .
$$

By (5.14) and $\Delta_{\rho} \cap \mathcal{R}=\emptyset$ it is easy to see that

$$
R^{*}=\inf \left\{R \geq 0 \mid\left(\frac{1}{1+R} \Delta_{\rho}+\frac{R}{1+R} \mathcal{R}\right) \cap \mathcal{R} \neq \emptyset\right\} .
$$

Let

$$
\mathcal{D}^{*}=\frac{1}{1+R^{*}} \Delta_{\rho}+\frac{R^{*}}{1+R^{*}} \overline{\operatorname{co}}\left(\Delta_{\rho} \cup \mathcal{R}\right) .
$$

Let us associate with each $Z$ the following number

$$
r_{Z}=\inf \left\{R \geq 0 \mid \exists\left(Z_{1}, \tilde{Z}\right) \in \Delta_{\rho} \times \operatorname{co}\left(\Delta_{\rho} \cup \mathcal{R}\right), \frac{1}{1+R} Z_{1}+\frac{R}{1+R} \tilde{Z}=Z\right\}
$$


As discussed in Corollary 3.10 Cherny [9], $\mathcal{D}^{*} \cap \mathcal{R}$ consists of all points in $\mathcal{R}$ with minimum $r_{Z}$. Following previous discussions, it is now clear that

$$
r_{Z}=\inf \left\{R \geq 0 \mid \exists\left(Z_{1}, \tilde{Z}\right) \in \Delta_{\rho} \times \mathcal{R}, \frac{1}{1+R} Z_{1}+\frac{R}{1+R} \tilde{Z}=Z\right\}
$$

Now let us defin the following function

$$
\left\{\begin{array}{l}
d: \Delta_{\rho} \times \mathcal{R} \rightarrow[0,+\infty], \\
d\left(Z_{1}, Z\right)=\inf \left\{R \geq 0 \mid \exists \tilde{Z} \in \mathcal{R}, \frac{1}{1+R} Z_{1}+\frac{R}{1+R} \tilde{Z}=Z\right\} .
\end{array}\right.
$$

Geometrically $d\left(Z_{1}, Z\right)$ can be justifie as follows: we connect $Z_{1}$ to $Z$ and continue until hitting the last point in $\mathcal{R}$, denoted by $\tilde{Z}$ (since $\mathcal{R}$ is $\sigma\left(L^{q}, L^{p}\right)$-compact, the last point exists). There exists $R \geq 0$ such that $Z=\frac{1}{1+R} Z_{1}+\frac{R}{1+R} \tilde{Z}$. With the above notations $d\left(Z_{1}, Z\right)$ is equal to $R$, i.e. $d\left(Z_{1}, Z\right)=R$. In the case that the continuation of the semi line $\overrightarrow{Z_{1} Z}$ hits $\mathcal{R}$ only in $Z$ (i.e. $Z=\tilde{Z}$ ) we put $d\left(Z_{1}, Z\right)=+\infty$. In the following lemma we show that the function $d$ is lower semi-continuous

Lemma 3 The function d defined above is $\sigma\left(L^{q}, L^{p}\right)$-lower semi-continuous.

Proof To show that $d$ is $\sigma\left(L^{q}, L^{p}\right)$-lower semi-continuous we have to prove that the set $C_{a}$ define as

$$
C_{a}=\left\{\left(Z_{1}, Z\right) \in \Delta_{\rho} \times \mathcal{R} \mid d\left(Z_{1}, Z\right) \leq a\right\}
$$

is $\sigma\left(L^{q}, L^{p}\right)$-closed for every positive number $a \in[0, \infty]$. To this end, let $a$ be a number in $[0, \infty]$ and suppose that $\left\{\left(Z_{1}^{n}, Z^{n}\right)\right\}_{n}$ is a sequence in $C_{a}$, converging in $\sigma\left(L^{q}, L^{p}\right)$ to $\left(Z_{1}, Z\right) \in \Delta_{\rho} \times \mathcal{R}$. If $a=\infty$ obviously $\left(Z_{1}, Z\right) \in C_{\infty}$ and then $C_{\infty}$ is closed. The case $a=0$ is never applied since we are assuming that $\Delta_{\rho} \cap \mathcal{R}=\emptyset$. So let us suppose that $a \in(0,+\infty)$. For each $n$, by definition there exists $\tilde{Z}^{n}$ such that

$$
Z^{n}=\frac{1}{1+d\left(Z_{1}^{n}, Z^{n}\right)} Z_{1}^{n}+\frac{d\left(Z_{1}^{n}, Z^{n}\right)}{1+d\left(Z_{1}^{n}, Z^{n}\right)} \tilde{Z}^{n} .
$$

Since $d\left(Z_{1}^{n}, Z^{n}\right)$ is bounded by number $a$ and also since by (5.10) $\Delta_{\rho} \times \mathcal{R}$ is $\sigma\left(L^{q}, L^{p}\right)$ compact, there is a subsequence $\left\{n_{k}\right\}_{k=1}^{\infty}$ such that $d\left(Z_{1}^{n_{k}}, Z^{n_{k}}\right)$ and $\tilde{Z}^{n_{k}}$ converge respectively to a number $d$ in $[0, a]$ and $\tilde{Z} \in \mathcal{R}$. Now by tending $n_{k} \rightarrow \infty$ in (5.16) we have $\frac{1}{1+d} Z_{1}+\frac{d}{1+d} \tilde{Z}=Z$, which by definition yields $d\left(Z_{1}, Z\right) \leq d \leq a$.

As mentioned in previous discussions, by Corollary 3.10 Cherny [9] and Lemma 3 one can deduce that

$$
\mathcal{D}^{*} \cap \mathcal{R}=\left\{Z \in \mathcal{R} \mid \exists Z_{1} \in \Delta_{\rho}, d\left(Z_{1}, Z\right) \text { is minimal }\right\} .
$$

The members of the set $\mathcal{D}^{*} \cap \mathcal{R}$ are the discount factors for the No Better Choice pricing rule. But interestingly the members of this set are also minimal for $(\mathcal{R}, \preceq$ ) (see the next theorem) which by Theorem 4.2 leads us to a good choice of the risk recovery.

Theorem 5.3 All members of $\mathcal{D}^{*} \cap \mathcal{R}$ are minimal for $(\mathcal{R}, \preceq)$.

Proof Let $Z \in \mathcal{D}^{*} \cap \mathcal{R}$. By Lemma 3, there exists $Z_{1}^{\min } \in \Delta_{\rho}$ such that $d\left(Z_{1}^{\min }, Z\right)$ is minimum over $\Delta_{\rho} \times \mathcal{R}$. By definition of $d$, we know that there exists $\tilde{\tilde{Z}} \in \mathcal{R}$ such that

$$
Z=\frac{1}{1+d\left(Z_{1}^{\text {min }}, Z\right)} Z_{1}^{\text {min }}+\frac{d\left(Z_{1}^{\text {min }}, Z\right)}{1+d\left(Z_{1}^{\text {min }}, Z\right)} \tilde{Z}
$$




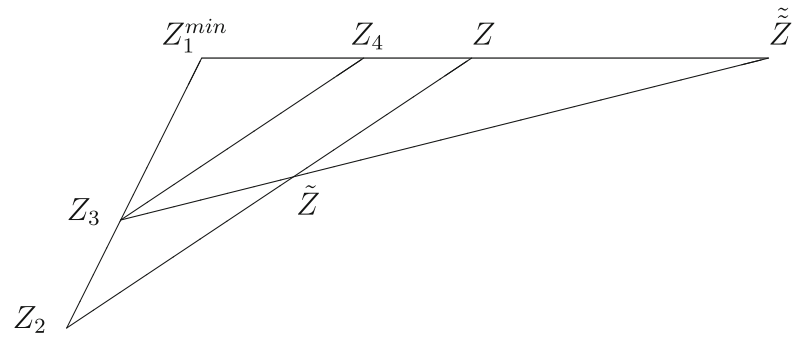

Fig. 1 The proof illustration of Theorem 53

Now let us suppose, to the contrary, that there exists $\tilde{Z} \in C(Z) \cap \mathcal{R}$ and $\tilde{Z} \neq Z$. By definition there exists $Z_{2} \in \Delta_{\rho}$ and $R \in[0,+\infty)$ such that $\frac{1}{1+R} Z_{2}+\frac{R}{1+R} Z=\tilde{Z}$. From this relation it turns out that $d\left(Z_{2}, \tilde{Z}\right) \leq R$ which yields $d\left(Z_{1}^{\min }, Z\right) \leq R<+\infty$. This assures us that $Z \neq \tilde{\tilde{Z}}$ (Fig. 1).

Since $Z$ is convex combination of $Z_{1}^{\min }, \tilde{\tilde{Z}}$ the three points $Z_{1}^{\min }, Z, \tilde{\tilde{Z}}$ are on the same direction. We claim that the point $\tilde{Z}$ cannot be on the line that passes through $Z_{1}^{\text {min }}, Z, \tilde{\tilde{Z}}$. In order to see this, firs note that since $\tilde{Z} \prec Z \prec \tilde{\tilde{Z}}$ we have that $\tilde{Z} \notin \vec{Z} \overrightarrow{\tilde{Z}}$. So if $\tilde{Z}$ is on the same direction as $Z_{1}^{\min }, Z, \tilde{\tilde{Z}}$ two possibilities remain: either $\tilde{Z} \in\left[Z_{1}^{\min }, Z\right)$ or $Z_{1}^{\min } \in[\tilde{Z}, Z)$. The firs is ruled out since obviously in that case $d\left(Z_{1}^{\min }, \tilde{Z}\right)<d\left(Z_{1}^{\min }, Z\right)$. The second possibility is also ruled out since in that case by convexity of $\mathcal{R}$, we get $Z_{1}^{\min } \in \mathcal{R}$.

Now we have four different points $Z_{1}^{\text {min }}, Z, \tilde{Z}, \tilde{Z}$ which are not in the same direction while three of them $Z_{1}^{\min }, Z, \tilde{\tilde{Z}}$ are. So the convex combination of these four points lie in a two dimensional affin space $P$. It is clear that $Z_{2} \in P$. Note that $Z_{2} \neq Z_{1}^{\text {min }}$, since otherwise $\tilde{Z}$ is on the the line passing through $Z_{1}^{\min }, Z, \tilde{\tilde{Z}}$. In the affin space $P$, the side $Z Z_{2}$ of the triangle $\Delta Z_{1}^{\text {min }} Z Z_{2}$ is hit by the semi-line $\overrightarrow{\tilde{Z}} \tilde{Z}$ in point $\tilde{Z}$. So the continuation should hit the other side $Z_{1}^{\text {min }} Z_{2}$ (the opposite side is impossible since again it puts $\tilde{Z}$ on the line passing through $Z_{1}^{\text {min }}, Z, \tilde{\tilde{Z}}$ ). Denote the hit point by $Z_{3}$. By convexity, $Z_{3}$ belongs to $\Delta_{\rho}$. On the side $Z_{1}^{m i n} Z$ of the triangle $\Delta Z_{1}^{m i n} Z Z_{2}$ we fin a point $Z_{4}$ such that $Z_{3} Z_{4}$ is parallel to $Z_{2} Z$. Obviously $Z_{4} \in\left(Z_{1}^{\min }, Z\right)$. Since $Z_{3} Z_{4}$ and $Z_{2} Z$ are parallel we have:

$$
\frac{\left|Z_{3} \tilde{Z}\right|}{|\tilde{Z} \tilde{Z}|}=\frac{\left|Z_{4} Z\right|}{|Z \tilde{\tilde{Z}}|}<\frac{\left|Z_{1}^{\text {min }} Z\right|}{|Z \tilde{\tilde{Z}}|}=d\left(Z_{1}^{\text {min }}, Z\right) .
$$

But by definition $d\left(Z_{3}, \tilde{Z}\right) \leq \frac{\left|Z_{3} \tilde{Z}\right|}{|\tilde{Z} \tilde{Z}|}$. Therefore, $d\left(Z_{3}, \tilde{Z}\right)<d\left(Z_{1}^{\min }, Z\right)$, which is a contradiction.

Acknowledgment This work is completed during the visit of Hirbod Assa from University of Carloss III of Madrid Hirbod Assa would like to thank University of Carloss III of Madrid and in particular Alejandro Balbás, for their great hospitality The authors would like also to thank the anonymous referees for their valuable comments This work is partially supported by University of Montreal (bourse de mobilité), the Spanish Government (Grant ECO2009-14457-C04), and the local Government of Madrid (Grant S2009/ESP-1594) 


\section{References}

1 Balbás, A, Balbás, R : Compatibility between pricing rules and risk measures: The CCVaR Revista de la Real Academia de Ciencias, Serie A, Matemáticas 103(2), 251-264 (2009)

2 Balbás, A, Balbás, R, Garrido, J : Extending pricing rules with general risk functions Eur J Oper Res 201(1), 23-33 (2010)

3 Balbás, A, Balbás, B, Heras, A : Optimal reinsurance with general risk measures Insurance Math Econom 44(3), 374-384 (2009)

4 Balbás, A, Balbás, R, Mayoral, S : Portfolio choice and optimal hedging with general risk functions: a simplex-like algorithm Eur J Oper Res 192(2), 603-620 (2009)

5 Björk, T, Slinkor, I : Towards a general theory of good-deal bounds Rev Finance 10(2), 221-260 (2006)

6 Cerný, A : Generalised sharpe ratios and asset pricing in incomplete markets Eur Finance Rev 7, 191-223 (2003)

7 Cerný, A, Hodges, S : Coherent risk measures on general probability spaces In: Mathematical Finance, Bachelier Congress 2000, pp 175-202 Springer, Berlin (2002)

8 Chan, T : Pricing contingent claims on stocks driven by Lévy processes Ann Appl Probab 9(2), 504-528 (1999)

9 Cherny, A S : Equilibrium with coherent risk Quantitative Finance Papers math/0605051, arXiv org, May 2006

10 Cochrane, J H , Saa-Requejo, J : Beyond arbitrage: Good deal asset price bounds in incomplete markets J Politic Econ 108, 79-119(2000)

11 Delbaen, F : Coherent risk measures on general probability spaces In: Advances in Finance and Stochastics, pp 1-37 Springer, Berlin (2002)

12 Delbaen, F : Risk measures for non-integrable random variables Math Finance 19(2), 329-333 (2009)

13 Filipovic, D, Svindland, G: The canonical model space for law-invariant convex risk measures is $L^{1}$ Mathematical Finance, forthcoming

14 Föllmer, H, Schied, A : Stochastic finance volume 27 of de Gruyter Studies in Mathematics Walter de Gruyter \& Co , Berlin (2002) extended edition, 2004 An introduction in discrete time

15 Jouini, E, Kallal, H : Arbitrage in securities markets with short-sales constraints Math Finance 5(3), 197-232 (1995)

16 Jouini, E, Kallal, H : Martingales and arbitrage in securities markets with transaction costs J Econom Theory 66(1), 178-197 (1995)

17 Jouini, E, Schachermayer, W, Touzi, N : Law invariant risk measures have the Fatou property In: Advances in Mathematical Economics vol 9, pp 49-71 Springer, Tokyo (2006)

18 Rockafellar, R T, Uryasev, S, Zabarankin, M : Generalized deviations in risk analysis Finance Stoch 10(1), 51-74 (2006)

19 Zalinescu, C : Convex Analysis in General Vector Spaces World Scientifi Press Co, London (2002) 